\title{
Real and reciprocal space electron tomography reveals structure and vacancies
}

Tom Willhammar ${ }^{1}$, Kadir Sentosun ${ }^{2}$, Stefanos Mourdikoudis ${ }^{3}$, Bart Goris ${ }^{2}$, Marnik Bercx ${ }^{2}$, Dirk Lamoen ${ }^{2}$, Bart Partoens ${ }^{2}$, Luis M. LizMarzan ${ }^{4}$, Sara Bals ${ }^{2}$, Gustaaf van Tendeloo ${ }^{2}$

${ }^{1}$ Department Of Materials And Environmental Chemistry, Stockholm, Sweden, ${ }^{2}$ EMAT, University of Antwerp, Antwerp, Belgium,

${ }^{3}$ Departamento de Quimica Fisica, Universidade de Vigo, Vigo, Spain, ${ }^{4}$ Bionanoplasmonics Laboratory, CIC biomaGUNE, San Sebastian, Spain

E-mail: tom.willhammar@mmk.su.se

Copper chalcogenides are known to find applications in different fields such as photonics, photothermal therapy and photovoltaics. Copper vacancies in the structure of these materials are a prerequisite for plasmonic activity, however an accurate understanding of the distribution of the defects and their influence on the properties still remains a challenge. One approach for studies of defects and disorders is total scattering methods and pair distribution function theories. In this work we present an alternative approach, based on electron microscopy. We demonstrate here that the structure of a novel phase of Cu1.5 $\pm x$ Te nanocrystals can be determined using electron diffraction tomography. In addition to that, real-space high-resolution electron tomography directly reveals the three-dimensional distribution of Cu vacancies in the structure. [1]

Recently, Li et al.[2] reported a new copper telluride nano-crystalline phase, displaying a novel crystalline phase and yielding interesting plasmonic properties toward sensing applications. The structure however has remained unknown so far due to its complexity. The nanocrystals has a cuboid morphology with a long edge of $\sim 30 \mathrm{~nm}$, see Figure $1 \mathrm{a}$.

In order to determine the atomic structure of the nanocrystalline material 3D electron diffraction methods were applied. A tilt series of selected area electron diffraction (SAED) patterns was acquired from a CuTe nanocrystal over a tilt range of -62.9 $\%+64.2^{\circ}$ with an tilt increment of $0.1^{\circ}$. This tilt series was combined into a 3D reconstruction of the reciprocal lattice, as shown in Fig. 1b. The reflections can be indexed in a cubic unit cell with an average cell parameter of $7.51 \AA$. Based on the intensities extracted from the reconstructed lattice an ab initio structure determination using direct methods resulted in a basic structure yielding $8 \mathrm{Te}$ atoms and 24 possible sites for $\mathrm{Cu}$ in one unit cell. Based on the composition half of the $\mathrm{Cu}$ sites must be absent. Further analysis of the 3D reciprocal lattice revealed reflections from a modulation along two directions as well as the presence of diffusely scattered intensities between the reflections.

In order to retrieve more details regarding the complex distribution of vacancies we used imaging methods and high resolution electron tomography. We carried out a tomographic reconstruction based on five high resolution HAADF-STEM images acquired with the rotation axis oriented along the short dimension of the cuboid shaped nanocrystal. Orthoslices through the reconstruction, perpendicular to the [100] and [010] directions, presented in Fig. 1c, enable the visualization of the distribution of vacancies in 3D. In this figure, the low intensity regions (blue) in between the Te atoms show the distribution of the $\mathrm{Cu}$ deficiencies. At this local scale, the order of the vacancies has a preference for a modulation of four average unit cells along one dimension of the nanocrystal. Furthermore, the reconstruction revealed the presence of 'channels' of $\mathrm{Cu}$ vacancies running through the material. A structure model was obtained based on the findings and DFT calculations of the dielectric properties shows good agreement with experimental optical properties.

[1] Willhammar, T et. al. Accepted Nature Communications (2017)

[2] Li, W. et al. J. Am. Chem. Soc. 135, 7098-7101 (2013)

a

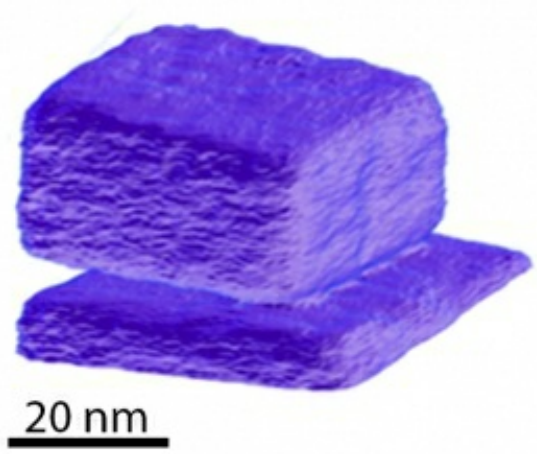

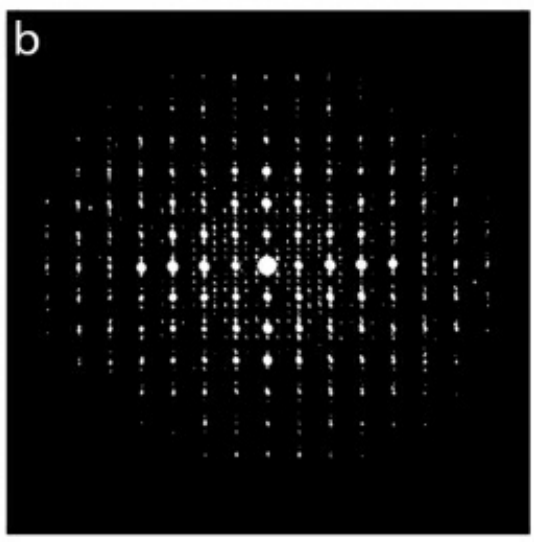

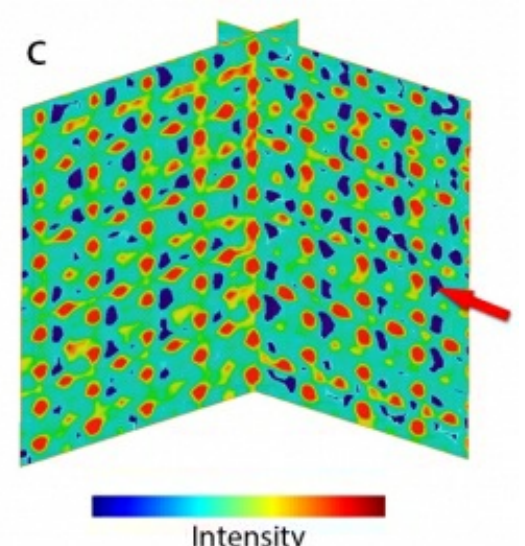

Intensity

Keywords: electron crystallography, electron tomography, vacancies 